\title{
Proton fixed-target scintillation experiment to search for millicharged dark matter
}

\author{
Kevin J. Kelly ${ }^{1, *}$ and Yu-Dai Tsai ${ }^{2,3, \dagger}$ \\ ${ }^{1}$ Theoretical Physics Department, Fermilab, P.O. Box 500, Batavia, Illinois 60510, USA \\ ${ }^{2}$ Fermilab, Fermi National Accelerator Laboratory, Batavia, Illinois 60510, USA \\ ${ }^{3}$ University of Chicago, Kavli Institute for Cosmological Physics, Chicago, Illinois 60637, USA
}

(Received 27 January 2019; published 29 July 2019)

\begin{abstract}
We propose a low-cost and movable setup to probe minicharged particles using high-intensity proton fixed-target facilities. This proposal, FerMINI, consists of a scintillator-based detector, requiring multicoincident scintillation signatures within a small time window, located downstream of the proton target of a neutrino experiment. During the collisions of a large number of protons on the target, intense minicharged particle beams may be produced via meson photo-decays and Drell-Yan production. We take advantage of the high statistics, shielding, and potential neutrino-detector-related background reduction to search for minicharged particles in two potential sites: the MINOS near detector hall and the proposed DUNE near detector hall, both at Fermilab. We also explore several alternative designs, including modifications to increase signal yield, and combining this detector technology with existing and planned neutrino detectors to better search for minicharged particles. FerMINI can achieve unprecedented sensitivity for minicharged particles in the $\mathrm{MeV}$ to few $\mathrm{GeV}$ regime with fractional charge $\varepsilon=Q_{\chi} / e$ as low as $10^{-4}$.
\end{abstract}

DOI: $10.1103 /$ PhysRevD.100.015043

\section{INTRODUCTION}

The quantization of electric charge has been one of the longest-standing mysteries in particle physics. The Standard Model (SM) $U(1)$ hypercharge group in principle allows arbitrarily small charges, yet experiments so far suggest that electric charge has a fundamental unit. This has inspired the concept of Dirac quantization [1] and motivated several considerations of grand unification theories [2,3]. The discovery of particles with electric charge less than the smallest quark charges, namely minicharged particles (MCP), would be a major paradigm shift. MCP have been studied and searched for on various fronts [4-16].

Given that MCP interact feebly with the SM particles through their small electric charge, they are also a potential solution to another well-established mystery of particle physics: dark matter. MCP may be a member of a dark sector [17] that couples to the Standard Model via a massless dark photon through kinetic mixing [18], and these or other dark sector particles may constitute the relic abundance of dark matter. Theories and signatures of dark

\footnotetext{
*kkelly12@fnal.gov

†tsai@fnal.gov
}

Published by the American Physical Society under the terms of the Creative Commons Attribution 4.0 International license. Further distribution of this work must maintain attribution to the author(s) and the published article's title, journal citation, and DOI. Funded by SCOAP. sectors have been heavily explored, especially in the context of neutrino and proton fixed-target experiments [19-33]. Recently, MCP as dark matter has been proposed to explain the anomalous $21-\mathrm{cm}$ hydrogen absorption signal reported by the Experiment to Detect the Global Epoch of Reionization Signature (EDGES) collaboration [34-36]. However, orthogonal constraints have been explored, and it has been demonstrated that the favored MCP candidates to explain the EDGES result cannot comprise the entirety of the observed relic dark matter abundance [37-39]. The favored range of masses of these $\mathrm{MCP}$ is below roughly a hundred $\mathrm{MeV}$, which is a region that could be explored in proton fixed-target experiments.

Probes of MCP and other weakly-interacting $\mathrm{MeV}-\mathrm{GeV}$ particles have been under intense study, due in large part to the fact that many dark matter and dark sector hypotheses fall into these categories. Additionally, experimental techniques to probe this region have matured significantly [17]. The most sensitive laboratory-based probes of MCP are threefold:

(i) Collider Probes

(ii) Electron Fixed-Target Experiments

(iii) Proton Fixed-Target and Neutrino Experiments

Both the Tevatron and Large Hadron Collider (LHC) have provided constraints on MCP for the first category $[6,11]$. Additionally, a dedicated experiment was specifically proposed to occupy the CMS P5 site to search for MCP $[13,14]$. Electron fixed-target experiments have been historically the most sensitive searches for MCP below 
$100 \mathrm{MeV}$. The dedicated SLAC MCP experiment [5,7] still provides leading sensitivity for MCP in this range. Several proposed electron-fixed target experiments (e.g., LDMX [40] and NA64 [41]) can further improve the sensitivity of MCP, but the mass reach would be limited by the beam energy. Finally, using neutrino experiments and protonson-fixed-targets to study MCP has been long proposed [8-10], but a dedicated analysis considering all the current and proposed near-future experiments has only been done recently [16], followed by the study based on reactor neutrino experiments for lower mass MCP [42].

We propose a Fermilab-based experiment to probe minicharged particles, FerMINI, that combines the techniques of dedicated searches at SLAC [5,7] and the LHC [13] with the advantages of neutrino facility sites [16]. We utilize the intense proton beams, for example, the existing Neutrinos at the Main Injector (NuMI) beamline and the future Long-Baseline Neutrino Facility (LBNF) beamlines, and place three groups of scintillator arrays downstream of the intense beam, shielded from strong electromagnetic radiation. The signature for $\mathrm{MCP}$ is the detection of one or a few photons produced when the particle traverses the scintillator, causing small ionizations and producing photons that generate photoelectrons (PE) in the photomultiplier tubes (PMT). We require such a detection in contiguous scintillator sets in each of the three detector groups in order to greatly reduce background. The two sites we explore are the existing Main Injector Neutrino Oscillation Search (MINOS) near detector hall and the proposed Deep Underground Neutrino Experiment (DUNE) near detector hall.

The nominal FerMINI setup substantially benefits from the large fluxes of MCP from the intense proton collisions. We will also discuss new ideas to combine the MCP detector with the neutrino detectors. FerMINI serves as an example to demonstrate that the proton-fixed target facilities could be natural habitats for the dedicated low-cost detectors to search for weakly interacting and long-lived particles.

We limit our attention to the assumption that the MCP are simply fermions with small $U(1)_{Y}$ hypercharges with masses between $\mathrm{MeV}-\mathrm{GeV}$ - constraints do not rely on the existence of dark photons nor assumptions of MCP abundance and velocity distributions in the local galaxy, but the bounds we derive serve as conservative constraints to the MCP-related dark matter and dark sector scenarios. Interestingly, finding $\mathrm{MCP}$ without an accompanying massless dark photon would have implications on not only GUT theories [2,3] but also string compactifications and quantum gravity [43]. The subtleties between different MCP scenarios are further explored in Refs. [44,45]. One can also use this setup to search for dark sector particles that couple to the SM through a light mediator, as demonstrated in Refs. [46,47]. Outside of probing MCPs and light dark matter scenarios, one can also utilize this proposal to probe the electric dipole moment of a heavy neutrino, as was proposed utilizing the milliQan facility [48].

Assuming MCP to be an appreciable fraction of the dark matter relic abundance, the parameter space FerMINI can probe falls into the regime of "strongly interacting dark matter" (see e.g. [49,50]). In this regime of the mass and cross section, the local dark matter flux would be strongly attenuated through the scattering with electrons and nuclei in the Earth's crust, atmosphere, and overburden, before reaching an underground dark matter direct-detection detector. As illustrated in [50], the dark matter in this parameter regime thus cannot be probed by any underground direct-detection experiments. Accelerator-based experiments like FerMINI that directly produce dark matter particles with high energy that can survive the attenuation through the propagation are thus needed to explore this parameter regime.

\section{MINICHARGED PARTICLE PRODUCTION}

We consider MCP $\chi$ with electric charge $Q_{\chi}$ and define $\varepsilon \equiv Q_{\chi} / e$. In proton fixed-target experiments, minicharged particles are produced via neutral meson decays and DrellYan processes:

Meson decays: We consider the following meson decays to the MCP $\chi$ :

(i) $\pi^{0} \rightarrow \gamma \chi \bar{\chi}\left(m_{\pi^{0}}=135 \mathrm{MeV}\right)$

(ii) $\eta \rightarrow \gamma \chi \bar{\chi}\left(m_{\eta}=548 \mathrm{MeV}\right)$

(iii) $J / \psi \rightarrow \chi \bar{\chi}\left(m_{J / \psi}=3.1 \mathrm{GeV}\right)$

(iv) $\Upsilon \rightarrow \chi \bar{\chi}\left(m_{\Upsilon}=9.4 \mathrm{GeV}\right)$

When produced in proton-proton collisions, each meson $\mathfrak{m}$ may decay into MCP with masses up to $m_{\mathfrak{m}} / 2$.

For $\mathfrak{m}=\pi^{0}, \eta$, the decay proceeds similar to that of $\mathfrak{m} \rightarrow \gamma e^{+} e^{-}$. We may write the total number of $\chi$ produced via these decays as [51,52]

$$
N_{\chi} \simeq 2 c_{\mathfrak{m}} \operatorname{Br}(\mathfrak{m} \rightarrow \gamma \gamma) \varepsilon^{2} \alpha_{\mathrm{EM}} N_{\text {POT }} \times I^{(3)}\left(\frac{M_{\chi}^{2}}{m_{\mathfrak{m}}^{2}}\right) .
$$

Here, $c_{\mathfrak{m}}$ is the number of meson $\mathfrak{m}$ produced per protonon-target (POT, total number $N_{\mathrm{POT}}$ ) in the target hall, and $I^{(3)}(x)$ characterizes the three-body decay ${ }^{1} \mathfrak{m} \rightarrow \gamma \chi \bar{\chi}$,

$$
I^{(3)}(x)=\frac{2}{3 \pi} \int_{4 x}^{1} d z \sqrt{1-\frac{4 x}{z}} \frac{(1-z)^{3}(2 x+z)}{z^{2}} .
$$

We find, using PYTHIA8 [53], $c_{\pi^{0}} \simeq 4.5$ and $c_{\eta} \simeq 0.5$ for $120 \mathrm{GeV}$ protons on target.

The $J / \psi$ and $\Upsilon$ mesons may decay directly via $\mathfrak{m} \rightarrow \chi \bar{\chi}$, and

$N_{\chi} \simeq 2 c_{\mathfrak{m}} \operatorname{Br}\left(\mathfrak{m} \rightarrow e^{+} e^{-}\right) \varepsilon^{2} N_{\mathrm{POT}} \times I^{(2)}\left(\frac{M_{\chi}^{2}}{m_{\mathfrak{m}}^{2}}, \frac{m_{e}^{2}}{m_{\mathfrak{m}}^{2}}\right)$,

\footnotetext{
${ }^{1}$ With $\varepsilon=1$, the product $\operatorname{Br}\left(\pi^{0} \rightarrow \gamma \gamma\right) I^{(3)}\left(m_{e}^{2} / m_{\pi^{0}}^{2}\right)$ reproduces the observed branching fraction of $\pi^{0} \rightarrow \gamma e^{+} e^{-\pi^{0}}$.
} 

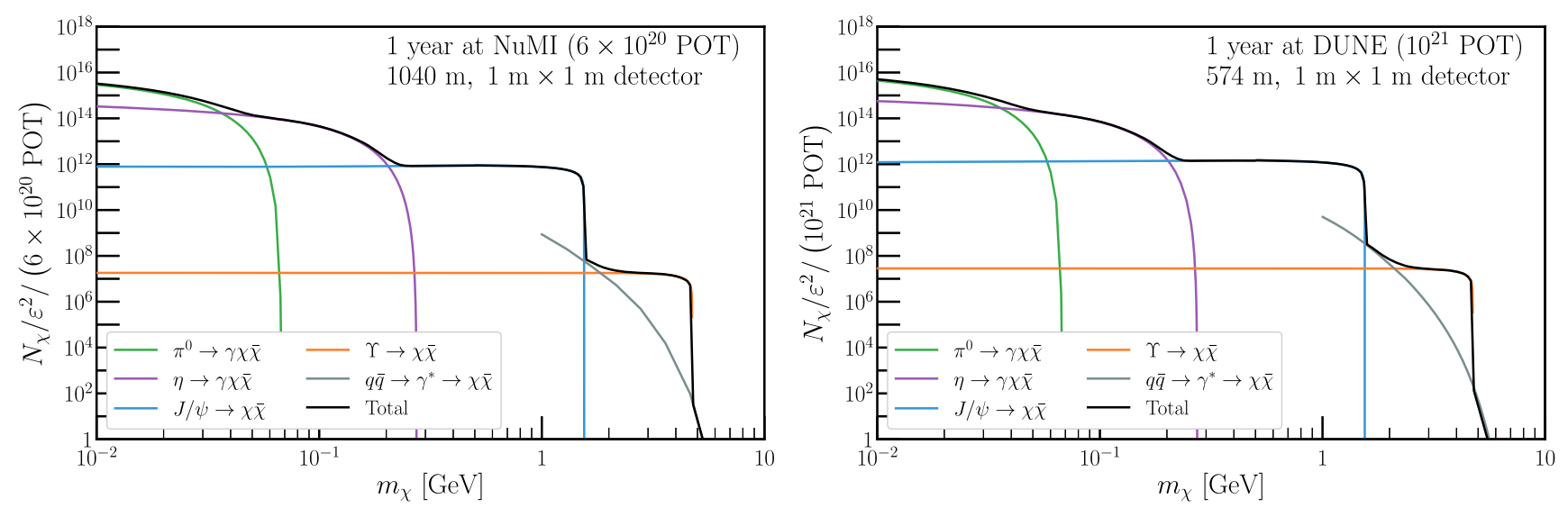

FIG. 1. Left (right) panel: expected number of MCP to reach a $1 \mathrm{~m} \times 1 \mathrm{~m}$ detector in the MINOS (DUNE) near detector hall, a distance of $1040 \mathrm{~m}(574 \mathrm{~m})$ from a $120 \mathrm{GeV}$ proton beam hitting a target. We assume 1 year of data collection, or $6 \times 10^{20}\left(10^{21}\right)$ protons on target. See text for detail.

where

$$
I^{(2)}(x, y)=\frac{(1+2 x) \sqrt{1-4 x}}{(1+2 y) \sqrt{1-4 y}} .
$$

The amount of $J / \psi$ or $\Upsilon$ produced per POT is far smaller than that of $\pi^{0}$ and $\eta$, though their production is important for larger $m_{\chi}$. We find that $c_{J / \psi} \simeq 4.4 \times 10^{-5}$ and $^{2} c_{\Upsilon} \simeq 2.5 \times 10^{-9}$.

Drell-Yan production: We also take into account $q \bar{q} \rightarrow$ $\chi \bar{\chi}$ using Martin-Stirling-Thorne-Watt (MSTW) parton distribution functions [54]. For the $120 \mathrm{GeV}$ protons on target for the NuMI and DUNE beams, this process is subdominant to meson-production, except for when $m_{\chi}>$ $m_{\Upsilon} / 2$ (see gray curve in Fig. 1). The Drell-Yan production curve is cut for $m_{\chi} \lesssim 1 \mathrm{GeV}$ since the MSTW parton distribution functions [54] are no longer appropriate below this energy.

While most of the $\chi$ particles produced via meson decay and Drell-Yan production are forward-going, not all pass through the $1 \mathrm{~m}^{2}$ cross-section of the detector at a distance of $\sim 500-1000 \mathrm{~m}$ away. We take this into account during our simulation and find that $\mathcal{O}\left(10^{-4}\right)$ of $\chi$ produced reach the detector.

\section{NOMINAL FERMINI SETUP AND SIGNATURE}

We propose to place a scintillator-based detector downstream of a proton beam to detect the MCP flux from the collisions of protons on a fixed target. In order to accumulate a large flux of MCP, the MCP detector will

\footnotetext{
${ }^{2}$ Since $c_{\Upsilon}$ is too small to generate $\Upsilon$ particles via Monte Carlo, we assume the flux of $\chi$ from this decay is similar to that from $J / \psi$ decay, extending the maximum allowed $m_{\chi}$ up to $m_{\Upsilon} / 2$ (instead of $m_{J / \psi} / 2$ ), scaled by the fraction $c_{\Upsilon} / c_{J / \psi}$. This scaling fraction is determined in Ref. [16] and the reference therein.
}

be downstream of a beam of a neutrino facility, specifically the existing NuMI or the upcoming LBNF beamline at Fermilab. We provide some detail of the two options here: the MINOS near detector hall is $1040 \mathrm{~m}$ downstream of the NuMI target hall, shielded by the absorber ( $840 \mathrm{~m}$ downstream) and $200 \mathrm{~m}$ of rocks [55]. The LBNF/DUNE ${ }^{3}$ setup is largely similar: a distance of $574 \mathrm{~m}$ from target to detector, with similar shielding $[53,56]$.

The nominal detector design of FerMINI directly follows Refs. [13,14], consisting of three stacks of scintillator arrays coupled to PMTs for readout. The physical observation for an MCP interacting with the detector is a very small amount of ionization and scintillation light. Based on Refs. [13,14,57], a particle with charge $Q_{\chi}=e$ traveling through a $\rho_{\text {scint. }}=1 \mathrm{~g} / \mathrm{cm}^{3}$ density scintillator deposits $2 \mathrm{MeV}$ per $\mathrm{cm}$ of travel. With a length of $1 \mathrm{~m}$, this results in roughly $200 \mathrm{MeV}$ of energy deposited. Such scintillators have a yield of about $10^{4}$ photons per $\mathrm{MeV}$, corresponding to $\mathcal{O}\left(10^{6}\right)$ photons produced. The amount of energy deposited, and therefore the number of photons produced in the scintillator, scales with $\varepsilon^{2}$, so we can expect sensitivity to reach $\varepsilon \sim 10^{-3}$ if single-photon readout is achievable. Each scintillator stack will consist of 400 $5 \mathrm{~cm} \times 5 \mathrm{~cm} \times 80 \mathrm{~cm}$ scintillator bars coupled to PMTs. Each bar will be oriented in the direction of the neutrino beam. A triple coincidence in all three adjacent scintillator bar-PMT sets in a small time window (nominally 15 nanoseconds) is required as an experimental signature to reduce the background.

We consider a runtime corresponding to one year of live-trigger time, corresponding to either $6 \times 10^{20} \mathrm{POT}$ for the NuMI beam [55] or $10^{21}$ POT for the DUNE beam [58]. This live-trigger time is not the same as the actual runtime, which would be slightly longer, however one

\footnotetext{
${ }^{3} \mathrm{We}$ assume that the LBNF beamline consists of $120 \mathrm{GeV}$ protons on target.
} 
year should be easily obtained, given the small dead/ live ratio estimated by milliQan [14] and the fact that NuMI will run for roughly five more years and LBNF is expected to run for more than ten years. This time also corresponds to the milliQan proposal using the High-Luminosity LHC (HL-LHC), allowing for direct comparison of sensitivity.

The advantage of having three separate scintillator array stacks is that, by requiring the observation of a signal in each stack in a narrow time window, we can drastically reduce the number of most background events [13,14]. If we define $\bar{N}_{\mathrm{PE}}$ as the average number of photoelectrons collected by the PMT due to a single minicharged particle $\chi$ passing through one stack of scintillators, then the probability of observing a single particle generating a signal in each stack, using a Poisson distribution, is

$$
P=\left(1-e^{-\bar{N}_{\mathrm{PE}}}\right)^{3} .
$$

We can roughly estimate $\bar{N}_{\mathrm{PE}}$ as follows: $\bar{N}_{\mathrm{PE}} \sim$ $\rho_{\text {scint. }} L_{\text {scint. }}\left\langle-\frac{d E}{d x}\right\rangle \times y \times E_{\text {det. }}$, where $\rho_{\text {scint. }}$ and $L_{\text {scint. }}$ are the scintillator density and the single-scintillator-bar length, respectively, $\left\langle-\frac{d E}{d x}\right\rangle$ is the mean rate of energy deposition (or energy loss) by a charged particle in the material, $y$ is the photon yield (number of photons per keV of energy deposited) of the scintillator, and $E_{\text {det. }}$ is the detection efficiency (the fraction of produced photons that generate PEs in the PMT) [59]. The mean energy deposition rate $\left\langle-\frac{d E}{d x}\right\rangle$ is proportional to $\varepsilon^{2}$. Strictly speaking, $\bar{N}_{\mathrm{PE}}$ is not exactly linearly proportional to $L_{\text {scint. }}$ and $\left\langle-\frac{d E}{d x}\right\rangle$. First, one needs to take into account the scintillator's attenuation length (roughly $2 \mathrm{~m}$ for the plastic scintillator) and $E_{\text {det. }}$. decreases as the scintillator length extends. Second, the dependence of $\bar{N}_{\mathrm{PE}}$ on $\left\langle-\frac{d E}{d x}\right\rangle$ should also be modified according to Birks' law [60].

We assume the scintillator is a Saint-Gobain BC-408 plastic scintillator and the PMTs are Hamamatsu R329-02 PMTs, as in Ref. [14] in performing calculations of these quantities, but specific scintillator and PMT choices can be later optimized for actual installation. We define $\xi$ by $\bar{N}_{\mathrm{PE}}=$ $(\varepsilon / \xi)^{2}$ and find $\xi \sim 2 \times 10^{-3}$ for the $m_{\chi}$ range of interest, in agreement with Ref. [14]. In our sensitivity estimates, we allow $\bar{N}_{\text {PE }}$ to vary depending on the mass/energy of $\chi$.

Given the much larger flux of MCP produced in a proton fixed-target facility compared to that by the LHC for MCP below $\sim 10 \mathrm{GeV}$ [16], one can potentially probe MCP with fractional charges one-order below $\varepsilon \sim 10^{-3}$. This advantage, in turn, brings up two complications: first, one should consider the most probable energy loss instead of the average energy loss in this case [59]. Second, one would be sensitive to small enough values of $\varepsilon$ that the expected number of photons produced per MCP passing through the detector is less than one, pushing the limit of the scintillator itself.

\section{BACKGROUND AND SENSITIVITY}

We will separate the discussions of the background into two categories: detector background and beam-related background.

First, we explore detector backgrounds. The background-reduction strategies developed in $[13,14]$ can be applied to the FerMINI detector and readout. Three major backgrounds: dark currents in the PMT, cosmic rays, and PMT after-pulses, and the reduction strategies are listed and discussed below.

(i) Requiring triple coincidence within $15 \mathrm{~ns}$ as a detection signature reduces all background sources except charged SM particles traversing the detector.

(ii) Offline vetoes of large-PE events allow for vetoing cosmic ray muons, etc.

(iii) Offsetting the middle detector array vetoes skimming tracks, which only deposit small numbers of PE.

(iv) A deadtime veto of after-pulses, by vetoing any small-PE depositions within $\sim 10^{-5} \mathrm{~s}$ of a large-PE deposition, can reduce backgrounds induced by SM particles.

After these background reductions, the largest remaining background would be the triple coincidence dark current counts in the PMT. As in Ref. [14], we assume a (conservative) dark current rate of $500 \mathrm{~Hz}$. With a triplecoincidence window of $15 \mathrm{~ns}$, this corresponds to roughly 300 background events per year.

Here we discuss backgrounds that are related to the intense proton beams: SM charged particles that reach, or are being produced in, the detector, and neutral particles (neutrinos and neutrons). Charged particles can be produced directly in the beam, through secondary production in the rock, or via particles scattering in the detector, potentially generating triple-coincidence events. The SM charged-particle flux in the beam would be shielded by the layers of rocks and monitored by the muon monitors before reaching the detector. Particles that arrive at or are produced in the detector would then be vetoed the techniques as discussed above. Production from hard neutrino and neutron scattering would also be vetoed as large-PE events, but soft collisions (e.g., neutrino neutral-current scattering) could mimic the low-PE ionization signature. We expect $\mathcal{O}\left(10^{4}\right)$ of such scatters in one year [58,61], which corresponds to $\mathcal{O}\left(10^{-18}\right)$ triple-coincidence background events for our MCP search. Overall, we assume the beam-related backgrounds will be of the same order as the detector backgrounds (300 events per year).

Additionally, neutrons produced by cosmic-ray muons or neutrinos from the beam could reproduce the soft scattering signal of MCP. We expect that the background rate due to cosmic rays will be small because of the depth of the detectors, and that the beam-related neutron backgrounds may be suppressed by using information of the timing of the neutrino beam. Additionally, requiring triple incidence 

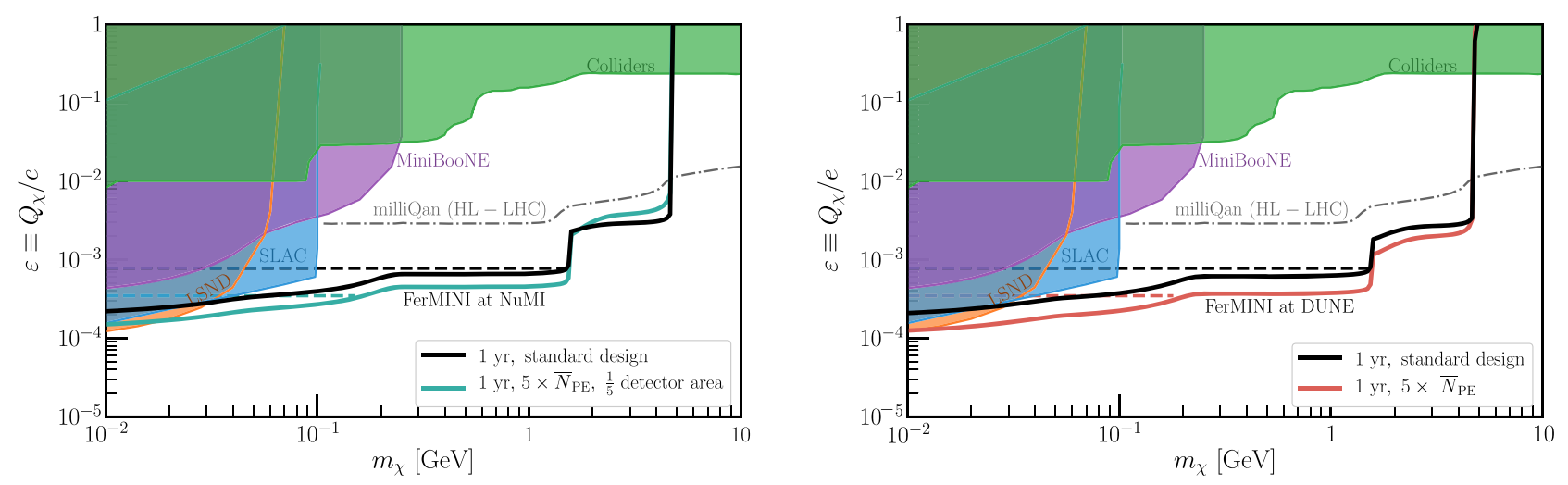

FIG. 2. Expected 95\% CL sensitivity to minicharged particles in the two potential sites we consider. Solid curves: sensitivity reach of FerMINI with one year of data collection. Colored curves correspond to modified detector design. We compare against the milliQan HLLHC reach [13,14], as well as existing constraints from SLAC [5], collider [6], MiniBooNE and LSND [16]. See text for more detail.

of such events, and the fact that they must occur in adjacent scintillator bars, should suppress this further. The possibility of an additional layer of scintillator (and requiring quadruple coincidence) would effectively make this background zero. We leave a more complete simulation of neutron-related backgrounds to future work.

A realistic determination of the beam-related background at Fermilab beams would require a detailed simulation including the beam production and the detector configuration. This should be possible with assistance from the NuMI-MINOS and LBNF-DUNE collaborations. We also note that the movable DUNE PRISM detector concept can aide in measuring beam-related backgrounds [62].

In Fig. 2 left and right we show 95\% CL sensitivity reaches assuming aforementioned background events and 1 year of live-trigger time at NuMI and DUNE, respectively. We also show alternative FerMINI designs with larger numbers of photoelectrons that lead to improved sensitivity.

Given the large flux of MCP at neutrino beams, we consider adding an additional stack of scintillators and requiring quadruple coincidence to further cut down the background. We find similar sensitivity reach to that in Fig. 2, however this would constitute a nearly zero-background search, an intriguing possibility.

\section{A. Alternative detector design with neutrino detectors}

Since the detector will be located inside existing/future neutrino near detector halls, we discuss the possibility of utilizing the neutrino detectors to better enhance our sensitivity. One idea would be to place FerMINI directly in front of or behind a neutrino detector and using the liquid argon technology to provide extra information on particles traversing the $\mathrm{MCP}$ detector and provide a veto on SM particles that could fake an MCP signal. In addition, one can look for mixed signature combining the scintillation signature discussed in this work and the hard electron scattering [16].
In particular, the excellent resolution of liquid argon could also be leveraged, by splitting the FerMINI detector arrays, placing some in front of and some behind the neutrino detector. MCP particles traveling through liquid argon can scatter off electrons, leading to single-electron events $[16,63]$. The combination of this signature with the scintillation signal of FerMINI could potentially further improve the MCP sensitivity. Both the NuMI and DUNE locations could provide such a combination, using the existing ArgoNeuT detector [64] and the upcoming DUNE near detector. At the DUNE site, the proposed 3D Scintillator Tracker could also be included in these combinations.

\section{DISCUSSION AND CONCLUSION}

Compared to milliQan, our setup consists of a much larger flux of MCP reaching the detector, due to the higher proton beam intensity. We find that FerMINI is sensitive to $\varepsilon$ below $10^{-3}$ and has better sensitivity than the milliQan search with the HL-LHC up to about $m_{\chi} \sim 5 \mathrm{GeV}$. The MCP flux is so large that it potentially saturates the scintillation limit and force us to consider modified detectors to fully explore the potential of FerMINI.

Reference [63] recently explored the capability of the existing ArgoNeuT and future DUNE Near Detector sensitivity to MCP by requiring multiple scatters in the liquid argon of the detector, a strategy that will significantly reduce the number of background events in such detectors. We find that, in comparison with Ref. [63], one year of FerMINI sensitivity places stronger constraints on $\varepsilon$ than ten years of data collection using the DUNE near detector for all masses $m_{\chi} \lesssim 4 \mathrm{GeV}$. In general, the one-year FerMINI sensitivity is a factor of $\sim 2-10$ times stronger than the ten-year DUNE sensitivity in Ref. [63]. An inexpensive detector like FerMINI, while dedicated to MCP searches, will provide extremely powerful sensitivity, and, as discussed in Section IVA, the combination of FerMINI and the liquid argon detectors could provide even stronger limits on MCP. 
Another advantage of FerMINI is that the NuMI beam is currently under operation. The LHC is under a long shutdown and milliQan has to wait for the LHC upgrades to complete in roughly two years to resume operation. The NuMI beam will be shut down in roughly five years, so it is important to initiate the FerMINI construction as soon as possible to take full advantage of the NuMI beam operation.

In addition, the great mobility of FerMINI makes it well suited for current and upcoming Fermilab neutrino experiments, where the detector can be used in the NuMI beam until its shutdown in roughly five years, then moved to the DUNE near detector hall. We regard the implementations of this proposal as a great opportunity for synergy between the collider, neutrino, and dark matter communities at Fermilab.

\section{ACKNOWLEDGMENTS}

We thank Joachim Kopp, Gabriel Magill, Zarko Pavlovic, Ryan Plestid, and Maxim Pospelov for useful discussions and input on various stages of completing this work. We especially thank Maxim Pospelov for the discussions of FerMINI alternative designs. We also thank members in the milliQan collaboration, specifically Albert De Roeck, Andy Haas, Christopher Hill, and Max Swiatlowski, for direct and indirect correspondence regarding the details of the milliQan proposal. We also thank Roni Harnik for pointing out the subtlety in determining the energy loss of a charged particle with very rare collisions in the detector, and Patrick Fox for correspondence regarding the NuMI beamline. We also thank Carlos Argüelles, Jeffrey Berryman, Kareem Farrag, Noah Kurinsky, Shirley Li, Mark Ross-Lonergan, Ibrahim Safa, and Gary Shiu for useful references and feedback on our draft. Y. T. thanks CERN, where this work was conceived, and its theory group for the hospitality. This manuscript has been authored by Fermi Research Alliance, LLC under Contract No. DE-AC02-07CH11359 with the U.S. Department of Energy, Office of Science, Office of High Energy Physics.

\section{APPENDIX: FIGURE ILLUSTRATION OF THE FERMINI EXPERIMENT}

In this section, we include a figure that illustrates the design of the FerMINI experiment. As shown in Fig. 3, one can see $\mathrm{MCP} \chi$ can be produced in the target through proton collisions, going through the existing experimental structure, and reach the FerMINI detector located downstream. The components of the figures are not to scale but serve as an illustration of the relative locations of the parts. We use part of the figures from [14] and [65] to make Fig. 3.

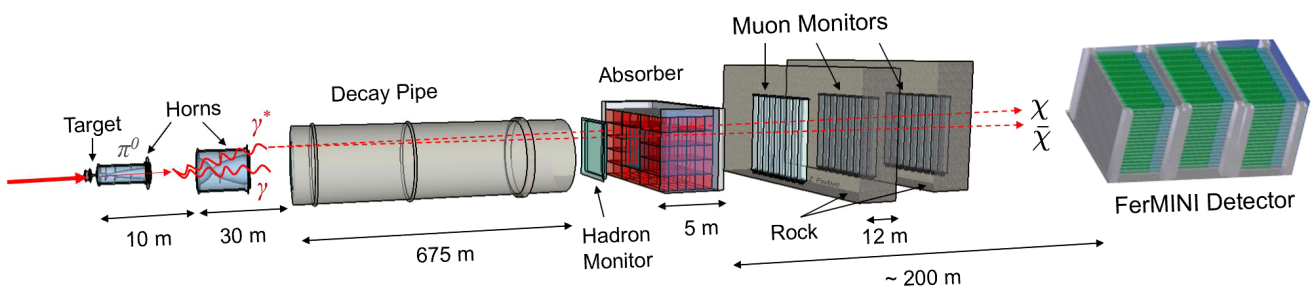

FIG. 3. An illustration of the FerMINI experiments utilizing the NuMI facility.

[1] P. A. M. Dirac, Quantized singularities in the electromagnetic field, Proc. R. Soc. A 133, 60 (1931).

[2] J. C. Pati and A. Salam, Unified lepton-hadron symmetry and a gauge theory of the basic interactions, Phys. Rev. D 8, 1240 (1973).

[3] H. Georgi and S. L. Glashow, Unity of All Elementary Particle Forces, Phys. Rev. Lett. 32, 438 (1974).

[4] M. I. Dobroliubov and A. Yu. Ignatiev, Millicharged Particles, Phys. Rev. Lett. 65, 679 (1990).

[5] A. A. Prinz et al., Search for Millicharged Particles at SLAC, Phys. Rev. Lett. 81, 1175 (1998).
[6] S. Davidson, S. Hannestad, and G. Raffelt, Updated bounds on millicharged particles, J. High Energy Phys. 05 (2000) 003.

[7] A. A. Prinz, Ph.D. thesis, Stanford University, 2001.

[8] E. Golowich and R. W. Robinett, Limits on millicharged matter from beam dump experiments, Phys. Rev. D 35, 391 (1987).

[9] K. S. Babu, T. M. Gould, and I. Z. Rothstein, Closing the windows on $\mathrm{MeV}$ Tau neutrinos, Phys. Lett. B 321, 140 (1994).

[10] S. N. Gninenko, N. V. Krasnikov, and A. Rubbia, Search for millicharged particles in reactor neutrino experiments: 
A probe of the PVLAS anomaly, Phys. Rev. D 75, 075014 (2007).

[11] S. Chatrchyan et al. (CMS Collaboration), Search for fractionally charged particles in $p p$ collisions at $\sqrt{s}=7$ TeV, Phys. Rev. D 87, 092008 (2013).

[12] R. Agnese et al. (CDMS Collaboration), First Direct Limits on Lightly Ionizing Particles with Electric Charge Less Than e/6, Phys. Rev. Lett. 114, 111302 (2015).

[13] A. Haas, C. S. Hill, E. Izaguirre, and I. Yavin, Looking for milli-charged particles with a new experiment at the LHC, Phys. Lett. B 746, 117 (2015).

[14] A. Ball et al., A letter of intent to install a milli-charged particle detector at LHC P5, arXiv:1607.04669.

[15] S. I. Alvis et al. (Majorana Collaboration), First Limit on the Direct Detection of Lightly Ionizing Particles for Electric Charge as Low as $e / 1000$ with the Majorana Demonstrator, Phys. Rev. Lett. 120, 211804 (2018).

[16] G. Magill, R. Plestid, M. Pospelov, and Y.-D. Tsai, Millicharged Particles in Neutrino Experiments, Phys. Rev. Lett. 122, 071801 (2019).

[17] J. Alexander et al., Dark sectors 2016 workshop: Community report, arXiv:1608.08632.

[18] B. Holdom, Two U(1)'s and epsilon charge shifts, Phys. Lett. 166B, 196 (1986).

[19] D. E. Brahm and L. J. Hall, U(1)-prime DARK MATTER, Phys. Rev. D 41, 1067 (1990).

[20] C. Boehm and P. Fayet, Scalar dark matter candidates, Nucl. Phys. B683, 219 (2004).

[21] M. Pospelov, A. Ritz, and M. B. Voloshin, Secluded WIMP dark matter, Phys. Lett. B 662, 53 (2008).

[22] J. D. Bjorken, R. Essig, P. Schuster, and N. Toro, New fixedtarget experiments to search for dark gauge forces, Phys. Rev. D 80, 075018 (2009).

[23] B. Batell, M. Pospelov, and A. Ritz, Exploring portals to a hidden sector through fixed targets, Phys. Rev. D 80, 095024 (2009).

[24] P. deNiverville, M. Pospelov, and A. Ritz, Observing a light dark matter beam with neutrino experiments, Phys. Rev. D 84, 075020 (2011).

[25] E. Izaguirre, G. Krnjaic, P. Schuster, and N. Toro, New electron beam-dump experiments to search for $\mathrm{MeV}$ to fewGeV dark matter, Phys. Rev. D 88, 114015 (2013).

[26] B. Batell, R. Essig, and Z. Surujon, Strong Constraints on Sub-GeV Dark Sectors from SLAC Beam Dump E137, Phys. Rev. Lett. 113, 171802 (2014).

[27] Y. Kahn, G. Krnjaic, J. Thaler, and M. Toups, DAEDALUS and dark matter detection, Phys. Rev. D 91, 055006 (2015).

[28] B. A. Dobrescu and C. Frugiuele, GeV-scale dark matter: Production at the main injector, J. High Energy Phys. 02 (2015) 019.

[29] P. Coloma, B. A. Dobrescu, C. Frugiuele, and R. Harnik, Dark matter beams at LBNF, J. High Energy Phys. 04 (2016) 047.

[30] P. deNiverville, C.-Y. Chen, M. Pospelov, and A. Ritz, Light dark matter in neutrino beams: Production modelling and scattering signatures at MiniBooNE, T2K and SHiP, Phys. Rev. D 95, 035006 (2017).

[31] M. Pospelov and Y.-D. Tsai, Light scalars and dark photons in Borexino and LSND experiments, Phys. Lett. B 785, 288 (2018).
[32] G. Magill, R. Plestid, M. Pospelov, and Y.-D. Tsai, Dipole portal to heavy neutral leptons, Phys. Rev. D 98, 115015 (2018).

[33] C. A. Argüelles, M. Hostert, and Y.-D. Tsai, Testing new physics explanations of MiniBooNE anomaly at neutrino scattering experiments, arXiv:1812.08768.

[34] J. D. Bowman, A. E. E. Rogers, R. A. Monsalve, T. J. Mozdzen, and N. Mahesh, An absorption profile centred at 78 megahertz in the sky-averaged spectrum, Nature (London) 555, 67 (2018).

[35] R. Barkana, Possible interaction between baryons and darkmatter particles revealed by the first stars, Nature (London) 555, 71 (2018).

[36] J. B. Muñoz and A. Loeb, Insights on Dark Matter from hydrogen during cosmic dawn, Nature (London) 557, 684 (2018).

[37] A. Berlin, D. Hooper, G. Krnjaic, and S. D. McDermott, Severely Constraining Dark Matter Interpretations of the 21-cm Anomaly, Phys. Rev. Lett. 121, 011102 (2018).

[38] R. Barkana, N. J. Outmezguine, D. Redigolo, and T. Volansky, Signs of dark matter at 21-cm? Phys. Rev. D 98, 103005 (2018).

[39] T. R. Slatyer and C.-L. Wu, Early-Universe constraints on dark matter-baryon scattering and their implications for a global 21 cm signal, Phys. Rev. D 98, 023013 (2018).

[40] A. Berlin, N. Blinov, G. Krnjaic, P. Schuster, and N. Toro, Dark matter, millicharges, axion and scalar particles, gauge bosons, and other new physics with LDMX, Phys. Rev. D 99, 075001 (2019).

[41] S. N. Gninenko, D. V. Kirpichnikov, and N. V. Krasnikov, Probing millicharged particles with NA64 experiment at CERN, arXiv:1810.06856.

[42] L. Singh et al., Constraints on millicharged particles with low threshold germanium detectors at Kuo-Sheng reactor neutrino laboratory, Phys. Rev. D 99, 032009 (2019).

[43] G. Shiu, P. Soler, and F. Ye, Milli-Charged Dark Matter in Quantum Gravity and String Theory, Phys. Rev. Lett. 110, 241304 (2013).

[44] Y.-D. Tsai, Ph.D. thesis, Cornell University, 2018.

[45] K. Kelly, R. Plestid, M. Pospelov, and Y.-D. Tsai (to be published).

[46] E. Izaguirre and I. Yavin, New window to millicharged particles at the LHC, Phys. Rev. D 92, 035014 (2015).

[47] A. Haas, https://web.fnal.gov/organization/theory/JETP/ 2016/Haas_milliQan_FermilabWC_9-8-2017.pdf.

[48] M. Sher and J. Stevens, Detecting a heavy neutrino electric dipole moment at the LHC, Phys. Lett. B 777, 246 (2018).

[49] A. Arvanitaki, S. Dimopoulos, S. Dubovsky, P.W. Graham, R. Harnik, and S. Rajendran, Decaying dark matter as a probe of unification and TeV spectroscopy, Phys. Rev. D 80, 055011 (2009).

[50] T. Emken, R. Essig, C. Kouvaris, and M. Sholapurkar, Direct detection of strongly interacting sub-GeV dark matter via electron recoils, arXiv:1905.06348.

[51] A. de Gouvêa, P. J. Fox, R. Harnik, K. J. Kelly, and Y. Zhang, Dark tridents at off-axis liquid argon neutrino detectors, J. High Energy Phys. 01 (2019) 001.

[52] V. De Romeri, K. J. Kelly, and P. A. N. Machado, Hunting on- and off-axis for light dark matter with DUNE-PRISM, arXiv:1903.10505. 
[53] T. Sjöstrand, S. Ask, J. R. Christiansen, R. Corke, N. Desai, P. Ilten, S. Mrenna, S. Prestel, C. O. Rasmussen, and P.Z. Skands, An introduction to PYTHIA 8.2, Comput. Phys. Commun. 191, 159 (2015).

[54] A. D. Martin, W. J. Stirling, R. S. Thorne, and G. Watt, Parton distributions for the LHC, Eur. Phys. J. C 63, 189 (2009).

[55] P. Adamson et al., The NuMI neutrino beam, Nucl. Instrum. Methods Phys. Res., Sect. A 806, 279 (2016).

[56] R. Acciarri et al. (DUNE Collaboration), Long-Baseline Neutrino Facility (LBNF) and Deep Underground Neutrino Experiment (DUNE), arXiv:1601.05471.

[57] C. Patrignani et al. (Particle Data Group), Review of particle physics, Chin. Phys. C 40, 100001 (2016).

[58] R. Acciarri and et al., Long-Baseline Neutrino Facility (LBNF) and Deep Underground Neutrino Experiment (DUNE) Conceptual Design Report Volume 2: The Physics Program for DUNE at LBNF, arXiv:1512.06148.

[59] M. Tanabashi et al. (Particle Data Group), Review of particle physics, Phys. Rev. D 98, 030001 (2018).
[60] J. B. Birks, Scintillations from organic crystals: Specific fluorescence and relative response to different radiations, Proc. Phys. Soc. London Sect. A 64, 874 (1951).

[61] R. Acciarri et al. (DUNE Collaboration), Long-Baseline Neutrino Facility (LBNF) and Deep Underground Neutrino Experiment (DUNE), arXiv:1512.06148.

[62] J. Cai et al., https://indico.fnal.gov/event/16205/contribution/ 2/material/0/0.pdf.

[63] R. Harnik, Z. Liu, and O. Palamara, Millicharged particles in liquid argon neutrino experiments, arXiv: 1902.03246.

[64] R. Acciarri et al. (ArgoNeuT Collaboration), Demonstration of $\mathrm{MeV}$-scale physics in liquid argon time projection chambers using ArgoNeuT, Phys. Rev. D 99, 012002 (2019).

[65] Z. Pavlovic, https://indico.cern.ch/event/4371/contributions/ 1087125/attachments/939323/1331996/zarko-nbi2006.pdf. 